\title{
Infective endocarditis late after patent foramen ovale percutaneous closure in an addict patient
}

\author{
Walter Vignaroli ${ }^{1}$, Angelo De Luca ${ }^{1}$, Fabio Capuano ${ }^{1}$, Francesca Boldini ${ }^{1}$, Euclide Tonelli $^{1}$, \\ and Giovanni Melina ${ }^{1}$ \\ ${ }^{1}$ Sant'Andrea Hospital
}

August 5, 2021

\begin{abstract}
We report an uncommon case of late infective endocarditis, in a patient with a history of intravenous drug abuse and HIV positivity, 9 years after a percutaneous closure of a patent foramen ovale. Despite a target and prolonged intravenous antibiotic therapy, he was then referred for surgical device and vegetation removal. Given the high risk associated with drug addiction lifestyle, especially in HIV positive cases, it is mandatory to observe this group of patients with lifelong follow-up and support. Furthermore, it may be considered a multidisciplinary-team approach to find the best option for these patients, also evaluating a surgical correction.
\end{abstract}

Infective endocarditis late after patent foramen ovalepercutaneous closure in an addict patient

Running Head: Amplatzer endocarditis in drug abuser

Walter Vignaroli $\mathrm{MD}^{1}$, Angelo De Luca $\mathrm{MD}^{1}$, Fabio Capuano $\mathrm{PhD}^{1 *}$, Francesca Boldini $\mathrm{MD}^{1}$, Euclide Tonelli $\mathrm{PhD}^{1}$, Giovanni Melina $\mathrm{PhD}^{1}$

${ }^{1}$ Department of Clinical and Molecular Medicine, Cardiac Surgery Unit, Sapienza University of Rome, Sant'Andrea Hospital, Rome, Italy

* Corresponding author

Conflict of interest: none

Source of founding: none

Consent: The patient gave written informed consent. The scientific ethics committee approved.

Data availability : The data that support the findings of this study are available from the corresponding author upon reasonable request.

\section{Corresponding author:}

Fabio Capuano, $\mathrm{PhD}$

Department of Clinical and Molecular Medicine, Cardiac Surgery Unit,

Sapienza University of Rome, Sant'Andrea Hospital,

Roma, Italy Via di Grottarossa 1035/1039, 00189

E-mail: fabiocapuano75@gmail.com

Tel.: +390633776743 
Fax.: +390633776317

Word Count : 858

Keywords: endocarditis, amplatzer, atrial septal defetc, cardiac surgery

\section{ABSTRACT}

We report an uncommon case of late infective endocarditis, in a patient with a history of intravenous drug abuse and HIV positivity, 9 years after a percutaneous closure of a patent foramen ovale.

Despite a target and prolonged intravenous antibiotic therapy, he was then referred for surgical device and vegetation removal.

Given the high risk associated with drug addiction lifestyle, especially in HIV positive cases, it is mandatory to observe this group of patients with lifelong follow-up and support. Furthermore, it may be considered a multidisciplinary-team approach to find the best option for these patients, also evaluating a surgical correction.

\section{MANUSCRIPT}

We describe a case of late infective endocarditis (IE), after percutaneous closure of a patent foramen ovale (PFO) in an intravenous drug abuser.

A 31-year-old man presented with worsening fever and cough associated with left knee swelling not responsive to steroid therapy. His past medical history included intravenous drug abuse and Human Immunodeficiency Virus (HIV) infection for which he was on anti-retroviral therapy. At the age of 22, he underwent percutaneous PFO closure with an Amplatzer device (AGA Medical, Golden Valley, Minnesota, USA) subsequent to an embolic stroke.

On physical examination, there was a widely harsh vesicular murmur with right basal hypophonesis, tachycardia and swelling on the right knee with functional impotence.

A Methicillin-sensitive Staphylococcus Aureus was isolated from blood cultures. Magnetic resonance and Computed Tomography examinations showed spots of infective emboli disseminated throughout the body, the lungs and multiple abscess collections with necrotic core in the left leg.

A trans-esophageal echocardiography was then performed (Fig.1A), showing multiple formations on the right side of the inter-atrial septum in an area corresponding to the previously implanted closure device and floating into the right atrium up to the tricuspid orifice (maximum size $5 \times 0.6 \mathrm{~cm}$ ). This large vegetation engaged in the tricuspid valve during each systole of the cardiac cycle. The left side of the inter-atrial septum appeared free from vegetations and fully covered by a thin layer of endothelium.

He was started on a target intravenous antibiotic therapy with piperacillin/tazobactam and teicoplanin, which was switched to daptomycin and amikacin due to persistent fever.

Considering the dimensions of the vegetation and the embolic events, the patient was then referred for cardiac surgery for Amplatzer@ removal and subsequent repair of the atrial defect.

Consent of the patients was obtained.

After a median sternotomy, cardiopulmonary bypass and cardioplegic cardiac arrest, an extensive vegetation of $4.5 \mathrm{~cm}$, including the previously implanted device (Fig.1B-C), was removed through a right atrial approach. The remaining inter-atrial defect was repaired with an autologous pericardial patch and operation completed in the usual fashion.

The postoperative course was uneventful, with regression of symptoms and progressive reduction of the disseminated abscess collections. The patient completed his course of antibiotic therapy and discharged home. At 6-months, he was free from symptoms and echocardiographic investigation was unremarkable. 
There are 26 cases of IE on atrial septal occluders described in literature. Out of those, 15/26 were positioned to repair Ostium Secundum defects and 11/26 for PFO's ${ }^{1}$.

Infective endocarditis is relatively rare after percutaneous inter-atrial septal defect closure but it can lead to several complications ${ }^{2}$ and It is reported to be more frequent early after the procedure ${ }^{1}$.

It has been previously described a percutaneous atrial septal defect closure performed in a 6 years old boy who eventually became a drug addict later in his life and developed an infective endocarditis which involved both the left and the right side of the septal occluder ${ }^{3}$.

The present case describes the late occurrence of infective endocarditis nine years after a percutaneous closure of PFO in an adult HIV positive drug abuser patient.

Considering the increase of drug abuse in developed countries, it is mandatory to observe this group of patients, with intense inpatient drug rehabilitation and psychological support, close and lifelong follow-up, customized treatment of other infectious diseases with a more aggressive approach and significant financial support in order to ensure patient therapy, accessibility and compliance.

In addition, given the high risk associated with drug addiction lifestyle, especially in HIV positive cases, it may be considered a multidisciplinary team approach to discuss a surgical approach, possibly minimally invasive, as an alternative to treat these defects and fully explain the available options to these patients.

Funding source: none

\section{BIBLIOGRAPHY}

1. Amedro P, Soulatges C, Fraisse A. Infective endocarditis after device closure of atrial septal defects: Case report and review of the literature. Catheter Cardiovasc Interv . 2017;89(2):324-334. doi:10.1002/ccd.26784

2. Verma SK, Tobis JM. Explantation of patent foramen ovale closure devices: A multicenter survey. JACC Cardiovasc Interv . 2011;4(5):579-585. doi:10.1016/j.jcin.2011.01.009

3. La Sala MS, Zohourian H, McKeown J, Snyder S. Late bacterial endocarditis in an intravenous drug user with an Amplatzer Septal Occluder. Texas Hear Inst J . 2020;47(4):311-314. doi:10.14503/THIJ-18-6903

\section{Figure 1. Echocardiographic and operative images}

Pre-operative trans-esophageal echocardiographic findings of the large vegetation (arrowheads) adherent to the body of the Amplatzer (AMP) projecting into the right atrium. RA: Right Atrium, RV: Right Ventricle. B) Surgical view of the vegetation (arrowheads) from right atrial approach. SVC: Superior Vena Cava, ICV Inferior Vena Cava and TV: Tricuspid Valve. C) Surgical sample of the vegetation (arrowheads) removed with the Amplatzer (AMP).

\section{Hosted file}

Endocardite su Amplatzer 600.pdf available at https://authorea.com/users/429241/articles/ 532977-infective-endocarditis-late-after-patent-foramen-ovale-percutaneous-closure-inan-addict-patient 